\title{
New generation wide-fast-deep optical surveys: petabytes from the sky
}

\author{
J. Anthony Tyson \\ Physics Department, University of California, Davis, CA 95616, USA \\ email: tyson@physics.ucdavis.edu
}

Many fundamental problems in optical astronomy - from planetary science, galactic structure, optical transients, to large-scale structure and cosmology - could be addressed though the same data set with millions of exposures in superb seeing, in multiple passbands, to very faint magnitudes over a large area of sky. This capability is largely driven by technology. In a logical progression towards this scientific capability, several increasingly ambitious wide-field optical surveys are planned in the next few years. A uniform high quality database covering all these science areas would be an ideal match to the VO. The above utopian goal of simultaneous pursuit of parallel surveys is achievable, but it relies on the ability to image a wide field quickly and deeply, and it is a non-linear function of the camera + telescope étendue.

A figure of merit for sky surveys is étendue, the product of aperture area in square meters and field of view in square degrees. The power of a survey facility to execute a given survey down to some limiting flux in selected wavelength bands over some area on the sky is proportional to its étendue. In practice one designs the survey strategy based on the étendue, in the context of the science driver(s). How does one decide on a overall survey strategy for a given facility? The science goals are often so different that they cannot be pursued simultaneously. Current surveys (as well as the upcoming PanSTARRS-1) optimize their survey strategy by pursuing different surveys mostly in series, giving them appropriate names like 'deep survey' 'wide survey' 'galactic plane survey' 'near-Earth object survey', etc., each with its own set of filters and observing cadence. However, there is in principle a thrshold in tendue above which is becomes increasingly possible to pursue multiple surveys in parallel. This multiplex capability occurs above about étendue $150 \mathrm{~m}^{2} \mathrm{deg}^{2}$ because the most demanding flux limit may be reached in a short time through standard filters. Breakthroughs in three areas of technology (large aspherical optics fabrication, microelectronics, and software) permit this major leap in capability. For the first time it will be possible to survey wide, fast, and deep simultaneously.

Data from a single active optics telescope with sufficient tendue can therefore address many scientific missions simultaneously. This is because short exposures also go deep, and with a large field of view the entire visible sky can be rapidly covered in multiple filters. The Large Synoptic Survey Telescope (LSST) with an tendue of 350 will provide unprecedented sky coverage, cadence, and depth. The 8.4 meter LSST and its 3.2 Gpixel camera and data system can attack high-priority scientific questions that are far beyond the reach of any existing or planned facility. The $20-30$ terabytes of data obtained each night will open a new window on the deep optical universe - the time domain enabling the study of variability both in position and time. This enables control of systematics required for precision probes of dark energy. Rarely observed events will become commonplace, new and unanticipated events will be discovered, and the combination of 
LSST with contemporary space-based and ground-based missions at other wavelengths will provide powerful synergies.

The LSST is a public-private consortium and the software and data will be open - a necessary condition for the effective operation of the VO links and access. It will be sited on Cerro Pachon in northern Chile, and commissioning is scheduled to begin in 2013. Up to 30000 square degrees of sky will be covered to $27.8 \mathrm{AB}$ mag $(5 \sigma)$. Six filter bands are planned: ugrizy covering the near UV to the near IR. This will enable photometric redshifts of 4 billion galaxies. The short $15 \mathrm{~s}$ exposures will enable unprecedented time domain studies. The unprecedented data volume must be processed immediately for both quality assessment and the science programs. Alerts on flaring or moving objects will be issued within seconds, and the database will be accessible through a data access center. Massively parallel astrophysics will result.

In each of these areas of astronomy, the full-hemisphere, high-time-resolution coverage of LSST will increase sample sizes by the largest factor ever achieved in optical astronomy, creating challenges and opportunities for the Virtual Observatory. 